\title{
Migration: Was wir nicht wissen. Perspektiven auf Forschungslücken
}

\author{
Christoph Reinprecht $\cdot$ Rossalina Latcheva
}

C) Springer Fachmedien Wiesbaden 2016

\section{Zu diesem Sonderheft}

Auch für das 21. Jahrhundert heißt es: Wir leben in einem Jahrhundert der Migration. Die gesellschaftliche Aufmerksamkeit für die Thematik ist international hoch, ebenso die Nachfrage an sozialwissenschaftlicher, insbesondere soziologischer Expertise. Das Spektrum an Tagungen, Symposien und Publikationen ist breit aufgefächert, und dies gilt nicht nur in thematischer Hinsicht, sondern auch in Bezug auf die Forschungsansätze, die vielfach interdisziplinär ausgerichtet sind. ${ }^{1}$

Aufgabe und Ziel von Sonderheften ist es in der Regel, über den neuesten Forschungsstand eines Spezialforschungsbereichs und dessen relevante Weiterentwicklungen und Herausforderungen zu berichten. Das vorliegende Sonderheft der Österreichischen Zeitschrift für Soziologie geht einen etwas anderen Weg. Die insgesamt neun Beiträge, die in diesem Heft versammelt sind, loten vor dem Hintergrund individueller Forschungserfahrung aus, was jene, die im Feld der migrationssoziologischen Forschung tätig sind, nicht wissen. Dass dieser Anspruch in einem einzelnen Heft nicht erschöpfend beantwortet werden kann, versteht sich von selbst: Zu ausdifferenziert ist die Forschungslandschaft, zu unübersichtlich das Feld der Wissensproduktion. Unsere Intention für dieses Heft ging aber von vorneherein über das

\footnotetext{
1 Vgl. etwa die mittlerweile 4. Jahrestagung zu Migrations- und Integrationsforschung in Österreich, die im September 2016 in Wien stattfand.

C. Reinprecht $(\bowtie)$

Institut für Soziologie, Universität Wien, Rooseveltplatz 2, 1090 Wien, Österreich

E-Mail: christoph.reinprecht@univie.ac.at

R. Latcheva

Agentur der Europäischen Union für Grundrechte (FRA), Schwarzenbergplatz 11, 1040 Wien, Österreich

E-Mail: Rossalina.LATCHEVA@fra.europa.eu
} 
Anliegen, in herkömmlicher Weise Forschungslücken aufzulisten, hinaus. Das Bestreben war vielmehr, die durch die Migrationsforschung selbst generierten blinden Flecken herauszuarbeiten, sei es als Folge spekulativer oder sogar antiwissenschaftlicher Ansätze, konzeptioneller und methodologischer Unstimmigkeiten oder des Forschungsprozesses selbst, der ja letztlich dafür verantwortlich ist, welche Ausschnitte sozialer Realität(en) Konturen erlangen oder auch unsichtbar bleiben bzw. gemacht werden.

\section{Migrationsforschung in der Sackgasse?}

Trotz ihrer Intensivierung und Ausdifferenzierung wird die Migrationssoziologie vielfach als blass und lückenhaft wahrgenommen, und sieht sich in zunehmendem Maße grundsätzlicher Kritik ausgesetzt. Ein Teil der Kritik richtet sich gegen den Charakter der Migrationsforschung als „Ausländerforschung“(Mecheril et al. 2013). Zum einen übernehme diese fraglos die Perspektive der Aufnahmeländer, in der Regel sei sie dann auch eher Integrations- als Migrationsforschung. Zum anderen bliebe sie selbst dann, wenn es ihr um Fragen sozialer Ungleichheit oder um die Untersuchung von Benachteiligung und Ausgrenzung geht, am dominierenden Ordnungs- und Integrationsparadigma orientiert. Doch woraus resultiert diese Problematik? Reflektiert sie das Erbe einer verengten nationalstaatlichen Konzeption? Nämlich Migration unter dem Gesichtspunkt von unidirektionalen Wanderungen (Aus- bzw. Einwanderung) zu begreifen, als bewusste Entscheidung für eine dauerhafte Verlegung des Lebensmittelpunktes, die im Wesentlichen einer ökonomischen Logik unterliegt (Wohlstandsgefälle, Einkommensdifferenzen, Arbeitsplatzchancen) und zugleich den Nationalstaat in seinem Bedürfnis nach Kontrolle und Steuerung der Bevölkerung herausfordert? Auch das grundsätzliche Dilemma sozialwissenschaftlicher Forschung, die differenziert und „leise“ argumentiert, wo der Aktualitätsbezug nach komplexitätsreduzierender Stellungnahme verlangt, könnte hier wirksam sein. Diskursiv aufgeladene Themen wie Flucht und Asyl, die Neuordnung der europäischen Grenzregime oder die Renaissance des (orientalistischen) Rassismus markieren die gesellschaftspolitischen Spannungsfelder, in denen sich die soziologische Migrationsforschung aktuell bewegt. Dieser Kontext fordert die Soziologie besonders heraus, da die von ihr vorgebrachten Fragestellungen, Diagnosen und Deutungen nicht unabhängig vom gesellschaftlichen und politischen Diskurs formuliert werden. Die im politischen und Alltagsdiskurs angebotenen Kategorien und Problemperspektiven (,mit Migrationshintergrund“, „Integration“, „zwischen den Stühlen“) durchdringen insofern auch die fachliche, wissenschaftliche Debatte. Darüber hinaus wirken in der Geschichte der Disziplin verankerte hegemoniale Perspektiven und Positionen, die im Kontext der Migrationsforschung als erkenntnishemmend wahrgenommen werden: Dazu zählen der seit vielen Jahren problematisierte „methodologische Nationalismus“, also die zentrale Stellung des Nationalstaats als sozialwissenschaftliche Denk- und Analysekategorie, sowie Eurozentrismus und Genderbias, d. h. die Standpunktbezogenheit der Forschung in Bezug auf als europäisch und männlich codierte Normen und Vorstellungen, oder der ökonomisch-funktionalistische Blickwinkel, der viele Forschungen nach wie vor 
wirkmächtig definiert (Pull-Push-Modelle, Rational-Choice basierte Analysen von Migrationsentscheidungen etc.). Diese Erbschaften werden aktuell intensiv diskutiert. Dies gilt besonders für den Zusammenhang von Nationalstaatsbildung, Migration und Sozialwissenschaften (vgl. Wimmer und Glick Schiller 2002; Beck und Grande 2010), aber auch für Eurozentrismus (Vollmer et al. 2015) und Genderbias (vgl. etwa Rodet 2008), die Übernahme vorherrschender normativer Orientierungen (vgl. Mecheril et al. 2013) oder den dominierenden Ökonomismus der Migrationsforschung (Castles 2010; Massey et al. 2008; Portes 2000).

Drei Problembereiche der Migrationssoziologie sind es, die in den Aufsätzen des Sonderhefts angesprochen und unter Bezug auf Literatur und empirische Forschung aufgearbeitet werden: Zum einen die gesellschaftstheoretisch oftmals unzureichende Rückbindung von Gegenstandsbenennung, Begrifflichkeit und Hypothesenbildung (,Migrationsforschung als Sackgasse?“). Zum zweiten die schwierige, weil zumeist auf mehr oder weniger impliziten heuristischen Vorannahmen beruhende Verwendung sozialer Kategorien („Eigenleben empirischer Kategorien“). Schließlich der selten systematisch untersuchte Beitrag der Forschung zur Produktion gesellschaftlicher (Un-)Sichtbarkeit („Forschung als Prozess von (Un-)Sichtbarmachung“). Es sind dies, wie wir meinen, Schlüsselfragen der aktuellen soziologischen Migrationsforschung.

\section{Zum Inhalt}

\subsection{Migrationsforschung als Sackgasse?}

In ihrer wohl allgemeinsten Definition untersucht Soziologie Migration als Ausdruck, Folge und Ursache von gesellschaftlichem Wandel, sei es auf der Mikro-, Meso- oder Makroebene (Portes 2010). Soziologisch gesehen interessiert nicht das demographische Ereignis der Wanderung selbst; vielmehr interessieren die sozialen Prozesse, die Wanderungen auslösen oder durch sie ausgelöst werden. Migration repräsentiert einen fait social total (Martiniello und Rea 2014, S. 3), insofern wir es mit gesellschaftlichen, teilweise auch institutionalisierten Praktiken zu tun haben. Migration fungiert hier als eine Triebfeder von Vergesellschaftung - ob im westafrikanischen Sahel mit seiner Tradition des saisonalen Wanderns, oder im globalen Norden als transnationale Pflegearbeit. Migrationssoziologische Forschung knüpft an diese basale Einsicht an. Sie fokussiert auf Veränderungen in der Sozialstruktur und deren Implikationen für das Verhältnis von Ordnung (Ungleichheitssystem), Konflikt (um Normen, Werte, Positionen) und Wandel (Transformation der Vergesellschaftungs- und Herrschaftsweisen). Migration und Integration, Migration und Konflikt, Migration und soziale Ungleichheit sind zentrale Verknüpfungen, mit denen die Migrationssoziologie operiert. Diese Verknüpfungen enthalten gesellschaftstheoretische Vorannahmen und sind normativ aufgeladen. Die zugrunde liegenden Menschen- und Gesellschaftsbilder sind nur selten Gegenstand der Literatur.

Sind es in der Chicago School, vor allem in Robert E. Parks (1939) publiziertem Modell eines „Race Relation Cycle“, Vorstellungen von Ethnizität, Wettbewerb und Assimilation, wobei das Einschmelzen in die Gesellschaft sich in erster Linie durch 
die Realität des Arbeitsmarktes vollzieht (Erfolg hat, wer sich dessen Regeln rasch anpasst), orientiert sich der kontinentaleuropäische Forschungskontext, insbesondere die deutschsprachige Soziologie, am Konzept der Unterschichtung (Privilegierung für vererbte Statuspositionen). Dies gilt auch für die inzwischen klassischen Studien wie jene von Hoffman-Nowotny (,Soziologie des Gastarbeiterproblems“, 1973), in der Migration als Beitrag zur Umverteilung von sozialen Spannungen zwischen Herkunfts- und Zielgesellschaften analysiert wird, oder Hartmut Essers „Wanderungssoziologie“ (1980) mit ihrem Fokus auf den Prozess der Eingliederung. Das Interesse für die Folgen der Unterschichtung, gerade auch langfristig, im Generationenübergang, sei es in Bezug auf die Persistenz systematischer Unterschiede und Ungleichheiten, von Diskriminierung und blockierter sozialer Aufwärtsmobilität oder die Herausbildung abgeschotteter, kastenähnlicher Strukturen, prägte lange Zeit den Mainstream der soziologischen Forschung, auch in Österreich. Bei all ihren großen Verdiensten besteht eine grundlegende (und verhängnisvolle) Charakteristik dieser Forschungen nicht nur darin, dass sie lange Zeit auf die Untersuchung der Arbeitsmigration der 1960er-Jahre fixiert blieb, sondern dass sie zumeist ohne systematische, theoretisch rückgebundene Analyse der historisch-politisch-ökonomischen Transformationsprozesse erfolgte ${ }^{2}$.

Vor fast zwei Jahrzehnten argumentierten Douglas Massey et al. in „Worlds in Motion“ (1998), dass neue Formen der Migration neue Theorien benötigen. Eines der zentralen Argumente der AutorInnen richtete sich damals gegen die (bis heute ungebrochene) Dominanz ökonomischer Migrationstheorien, die der zunehmenden Komplexität von Migration nicht gerecht würden und nicht-ökonomische Aspekte vernachlässigten (einen Ausgangspunkt bildete damals das wachsende transnationale Migrationsgeschehen zwischen Mexiko und den USA). Eine ungenügende gesellschaftstheoretische Fundierung wird der Migrationsforschung auch in zahlreichen aktuelleren Beiträgen attestiert, Stichworte sind Migration und soziale Transformation, methodologischer Transnationalismus, Grenzregime, Autonomie der Migration, postkoloniale und postmigrantische Positionen (siehe u. a. Amelina et al. 2015; Favell 2014; Martiniello und Rea 2014; O’Reilly 2012; Skeldon 2012; Tsianos und Karakayali 2010; Cvajner und Sciortino 2010; Haer 2010; Papadopoulos et al. 2008). Nach Stephen Castles (2010), der seit vielen Jahren eine Anbindung der Migrationsforschung an die sozialwissenschaftliche Theoriebildung einfordert und die Debatte mit transformationstheoretischen Überlegungen bereichert, verleiten die wachsende Diversität und Unvorhersagbarkeit von Migration ebenso zu Theorieferne wie eine zu große Politiknähe (Auftragsforschung), die den reflexiv-kritischen Umgang mit ontologischen, epistemologischen und/oder methodologischen Fragen hemmt. Der Kern der Problematik bestehe darin, dass sich der Mainstream der Migrationsforschung von den Entwicklungen der allgemeinen Gesellschafts- und Sozialtheorie entkoppelt habe. Neuere Forschungsansätze, die auf die Veränderung und Dynamisierung der zeitlichen und räumlichen Migrationsmuster reagieren und mit Begriffen

\footnotetext{
${ }^{2}$ Einen Überblick über die österreichische Forschungslandschaft geben der erste und zweite „Österreichische Migrations- und Integrationsbericht“ (Fassmann und Stacher 2003; Fassmann 2007) sowie die im zweijährigen Modus erscheinenden Jahrbücher „Migration und Integration - wissenschaftliche Perspektiven aus Österreich“ (Dahlvik et al. 2012, 2014; Carvill Schellenbacher et al. 2016).
} 
wie „transnational“, „spatial“ oder „,cosmopolitan turn“ und „mobility studies“ in Verbindung stehen, begünstigen im Prinzip eine solche Rückbindung, fordern jedoch die in Kategorien nationaler Gesellschaften denkende Soziologie heraus, nicht zuletzt aufgrund der eingeforderten Disziplinen übergreifenden (wenn nicht sogar post-disziplinären) Arbeitsweise (vgl. Amelina et al. 2013).

Braucht es anstelle einer Migrationssoziologie also eine gute Gesellschaftsanalyse, wie etwa Erol Yildiz (2015, S. 22) suggeriert? Auch die für dieses Heft verfassten Beiträge von Breda Gray, Kenneth Horvath und Claudia Vorheyer argumentieren für eine Überwindung migrationssoziologischer Engführungen. Der Beitrag von Kenneth Horvath untersucht am Beispiel Österreichs „Die unbekannten Pfade der Migrationspolitik“, indem er das Potential einer transformationsanalytischen Perspektive im Sinne Stephen Castles (2010) für die Analyse der Beziehungen zwischen migrationspolitischem Wandel und zeitgleich ablaufenden politisch-ökonomischen Veränderungsprozessen aufzeigt. Als Beispiel für die Verortung politischer Migrationsprozesse in breiteren gesellschaftlichen Verhältnissen dient der Bezug des Gastarbeiterregimes der 1960er und 1970er-Jahre auf das Inlandarbeitsschutzgesetz aus dem Jahr 1925, das erstmals eine rechtliche Differenzierung zwischen nationaler und migrantischer Arbeitskraft formulierte. Horvath geht der Frage nach, wo die politischen Instrumente herkamen, mit denen Gastarbeit in den 1960er Jahren sozialpartnerschaftlich verwaltet werden konnte. Er identifiziert ein Wechselspiel aus Sekuritisierung und Ökonomisierung der Migration in der konkreten gesellschaftlichen Konstellation und zeigt auf, wie dieses Wechselspiel zum Verständnis aktueller Entwicklungen und Steuerungsregime beitragen kann. Zudem zeigt dieser Beitrag, dass die aktuelle Verhandlung migrationspolitischer Fragen in die Transformation und Reproduktion sozialer Ungleichheitsverhältnisse eingebunden ist. Dieses Einbetten des Migrationsdiskurses in gesellschaftlich relevante Entwicklungen erlaubt es, die Entpolitisierung und Politisierung von Migration als Epochenphänomene zu interpretieren und nicht als Idiosynkrasien des österreichischen Nachkriegsarrangements.

Auch Breda Gray fordert in ihrem Aufsatz „Beyond Migration Studies“ eine Zusammenführung der Migrationsforschung und der soziologischen Forschung zu gesellschaftlichem Wandel und Transformation. Gray analysiert die drei interdisziplinären Zugänge - Transnational Studies, Diaspora Studies und Mobility Studies in Hinblick auf ihr Potential, Migration im Kontext sozialer Konflikte und von sozialem Wandel zu untersuchen. Zudem untersucht die Autorin, inwiefern diese drei Forschungsfelder den Kräften entgegenwirken, die eine Vereinnahmung des relevanten Migrationswissens anstreben. In ihrem Text „Transnational Mobiles“ versucht Claudia Vorheyer sozialwissenschaftliche Konzepte von Transnationalismus und Kosmopolitisierung für die Migrationssoziologie fruchtbar zu machen. Auf Grundlage eines qualitativ-biographischen Forschungsansatzes widmet sich Vorheyer dem in der Migrationsforschung bislang wenig beachteten Phänomen der transnational mobiles. Vorheyer beschäftigt die Frage, wie transnationale Mobilität subjektiv verarbeitet wird, insbesondere welche Bedeutung temporäre Wanderungen, bei denen zwei (oder auch mehr) alternative Lebensmittelpunkte aufrechterhalten werden, für die Alltagswelt und den Lebensverlauf der transnational mobiles haben. Dabei steht die Frage im Zentrum, inwieweit die mehrfachen transnationalen Migrationserfah- 
rungen mit Kosmopolitisierungsprozessen einhergehen und Veränderungen in den Zugehörigkeitsgefühlen und Identitätskonstruktionen bedingen.

\subsection{Eigenleben empirischer Kategorien}

Was wir nicht wissen, betrifft auch scheinbar Evidentes: „Although it is commonly believed that the volume, diversity, geographical scope, and overall complexity of international migration have increased as part of globalization processes, this idea has remained largely untested“ (Czaika und de Haas 2014, S. 283). Je nach verwendeter Datengrundlage kommt die Bevölkerungs- und Migrationsforschung zu unterschiedlichen Ergebnissen. Als dauerhafte, d. h. länger als einjährige Verlegung des Lebensmittelpunktes, die auch administrativ dokumentiert ist, nimmt Migration in absoluten Zahlen zu, allerdings bei eher konstantem Anteil an der Weltbevölkerung (vgl. dazu Abel und Sander 2014). Die Situation ändert sich bei Berücksichtigung von kurzfristigen Wanderungen (einschließlich von Pendel- und zirkulärer Migration) sowie von Fluchtmigration: in beiderlei Hinsicht haben die Dynamik und Komplexität des Wanderungsgeschehens erheblich zugenommen (Papastergiadis 2010). Sie variiert aber auch nach regionaler Perspektive: So sind zirkuläre Wanderungen und Fluchtmigration vor allem für Nachbarländer bedeutsam. Asien hat relativ geringe Anteile am internationalen Migrationsgeschehen, die Wanderungsmuster sind allerdings besonders komplex. Eine Intensivierung (und Diversifizierung) internationaler Wanderungen erleben hingegen die meisten Länder der Europäischen Union. In der Vorstellung, dass sich im Kontext der Globalisierung das Migrationsgeschehen insgesamt intensiviert und diversifiziert, manifestiert sich so gesehen eine tendenziell eurozentrische Sicht auf die Welt (Czaika und Haas 2014, S. 314).

Unsichere Datengrundlagen und uneindeutige, einander auch widersprechende Wissensbestände spiegeln eine zunehmend komplexe Realität des internationalen Migrationsgeschehens. Dieser Realität entsprechen nicht nur die heterogene Zusammensetzung, vielfältigen Formen und Dynamiken von Migration, sondern auch eine wachsende Unübersichtlichkeit der institutionellen Regelungen und Rahmenbedingungen. So erzeugen nationalstaatliche Regulierungsversuche immer kleinteiligere Kategorien von Zuwanderungs- und Aufenthaltsberechtigung, um auf diese Weise den Spielraum für Mobilität einzuengen und Bedingungen für das Migrationsmanagement abzustecken. Dies führt dazu, dass die administrativen Daten, die auf Grundlage dieser Regelungen gesammelt werden, aufgrund der unterschiedlichen Kategorien, schwer vergleichbar sind (Fassmann et al. 2009). Zugleich kollidieren die rechtlich zunehmend verregelten Migrationsregime mit der Nachfrage nach niedrig entlohnter, flexibel einsetzbarer und informell beschäftigter Arbeitskraft. $\mathrm{Ob}$ Saisonarbeit in der Landwirtschaft, Gastronomie oder Bauwirtschaft; über Subunternehmen organisierte Beschäftigung in Reinigung, Überwachung oder Zustellung; oder haushaltsnahe Dienstleistungen wie die Betreuung und Versorgung von Kindern oder Pflegebedürftigen: Migration bildet einen integralen Bestandteil einer neoliberal strukturierten, globalisierten Welt (Castles 2010, S. 1580 f.), die infolge der Kontrollregime häufig den Charakter von irregulärer Wanderung annimmt. Unter den geschätzten 14 Mio. Menschen, die jährlich weltweit wandern (,migration flow“), 
wird der Anteil der nicht dokumentierten Arbeitskräfte auf rund ein Fünftel geschätzt (vgl. Goldin et al. 2012, S. 124 ff.).

An der herkömmlichen Migrationsforschung wird kritisiert, dass sie dazu neigt, administrative Schemata und Kategorien zu übernehmen und unhinterfragt anzuwenden. Auf diese Weise liefere sie sich der Logik der nationalstaatlichen Migrationsregime aus und trage dazu bei, herrschende Differenzordnungen zu festigen (vgl. Dahinden 2016). Die Problematik ist vielschichtig. So bedient sich evidenzbasierte Politikgestaltung meist sozialwissenschaftlicher Daten, die Staatsbürgerschaft, Geburtsland oder Migrationshintergrund als Ordnungskriterien verwenden, wodurch in der Folge nicht nur die gesellschaftliche Diversität (Milieudifferenzierung) potentiell unterschätzt wird, sondern auch die Ursachen von sozialer Ungleichheit und Benachteiligung verschleiert werden können (Perchinig und Troger 2011). Zugleich geraten Forschungsfragen aus dem Blickfeld, die das Spannungsverhältnis von sozialer, symbolischer und physischer Sichtbarkeit (vgl. Brighenti 2007) zum Thema haben, etwa im Zusammenhang mit der politischen und öffentlichen Thematisierung von Flucht und Asyl oder der Unverhältnismäßigkeit polizeilicher und behördlicher Aufmerksamkeit gegenüber Personen, die sich sichtbar vom Mainstream unterscheiden, sei es wegen ihrer Hautfarbe, des Rufs des Wohnviertels oder der Kleidung, die Zugehörigkeit zu einer anderen Religiosität verrät - und ihrer „Unsichtbarkeit““ in allen relevanten administrativen und behördlichen Statistiken (von Registern und Zensus über Gesundheitswesen zu Kriminal- und Justizstatistik).

Dass empirische Kategorien ein mitunter problematisches Eigenleben entfalten, lässt sich auch am Beispiel der Kategorie „Drittstaatsangehörigkeit“ demonstrieren. Die Subsumierung unter dieses Label führt nicht nur zu einer Homogenisierung und Simplifizierung unzähliger Migrationsbiographien, die über unterschiedliche Wege nach Europa gelangen, unterschiedliche Ziele verfolgen, ihre Migrations-, Integrations- und Desintegrationerfahrungen täglich neu machen und ihre Migrations- als Lebensprojekte subjektiv interpretieren und evaluieren (Latcheva und Herzog-Punzenberger 2011), sondern schafft zugleich eine ,verwaltete Kategorie“ mit fremd zu- und festgeschriebenen Defiziten und Problemen der Integration. Während die etablierte semantische Praxis zwischen Mobilität und Migration unterscheidet und diese Begriffe unterschiedlich positiv bzw. pejorativ konnotiert, ist eine wachsende bürokratische Maschinerie damit beschäftigt, Migration abzuwickeln und die zugewanderte Bevölkerung nach rechtlich-administrativen Gesichtspunkten zu gruppieren, d. h. die Berechtigten von jenen, die als nicht berechtigt oder irregulär angesehen oder aus ökonomischen oder Gründen der inneren Sicherheit unerwünscht sind, herauszufiltern (Dahlvik und Reinprecht 2015, S. 45).

Drei Beiträge sind es, die sich in unserem Heft kritisch mit dem Eigenleben empirischer Kategorien befassen, die der Produktion von Erkenntnis und jeder aufgeklärtrationalen Politik zuwiderlaufen. In seinem Beitrag „Residentielle Segregation nach Nationalität“ beschäftigt sich Jens Dangschat mit dem widersprüchlichen Diskurs über Migration und residentielle Segregation. Er hinterfragt die weitverbreitete und ursprünglich innerhalb der humanökologischen Stadtsoziologie entwickelte These, wonach eine hohe Konzentration an AusländerInnen in einem Wohnquartier die Integration in die Aufnahmegesellschaft behindert, indem er nachweist, dass keine eindeutigen Belege für die Untermauerung dieser These existieren. Die Wider- 
sprüchlichkeit wissenschaftlicher Ergebnisse führt Dangschat auf eine bedenkenlose Übertragung sozialräumlicher Konzentrationen von Ort zu Ort, zu unterschiedlichen Zeitpunkten, bezogen auf unterschiedliche minorisierte Gruppen und zwischen unterschiedlichen Wohnungs(teil)märkten zurück. Seiner Meinung nach findet damit auch eine Übertragung sozialer Ungleichheit entlang von Nationalitäten, Ethnien oder dem Einkommen über die Gesetzmäßigkeiten des Wohnungsmarktes in den Siedlungsraum zurück - ein Prozess, der zeit- und raumunabhängig erscheint, indem der gesellschaftliche Kontext für die Erklärung sozialräumlicher Wandel kaum Beachtung findet. Dangschat stellt sich die Frage, wie ein ,gesichertes Wissen“ erarbeitet werden kann, sodass Stakeholder in Politik und Verwaltung beim Lösen sozialräumlicher Herausforderungen auf wissenschaftliche Erkenntnisse zurückgreifen können. Dabei geht der Autor dem Eigenleben empirischer Kategorien wie „Segregation“, „Nationalität“ und „Migrationshintergrund“ nach und hinterfragt ihre Verwendbarkeit für eine zweckmäßige Stadtplanung. Dangschat zeigt, dass die Segregationsforschung kaum auf Erkenntnisse der sozialen und räumlichen Ungleichheitsforschung Bezug nimmt - eine These, die die Argumentation von Kenneth Horvath und Breda Gray weiterspinnt. Zugespitzt schließt Dangschat seine Ausführungen mit der Forderung auf ein Recht auf ,,partielle“ Identifikation beziehungsweise „Nicht-Integration“", da ein souveräner Umgang mit wachsender Mobilität und damit einhergehender Anwesenheit an mehreren sozialen und geographischen Orten nur auf diese Weise möglich sei.

Der Beitrag von Walter Fuchs, Katrin Kremmel, Andrea Kretschmann und Arno Pilgram „Migration, Legalität und Kriminalität“ zeigt jene Bereiche rechtsoziologischen Nichtwissens innerhalb der Migrationsforschung auf, die im Schatten enormer Fülle an administrativem Wissen entstehen und bestimmte Kontroll- und Verwaltungspraktiken nach sich ziehen. Die AutorInnen plädieren für einen Perspektivenwechsel, von der Sicht, die MigrantInnen vorrangig als Objekte der Sicherheitsverwaltung begreift, hin zu einer Auffassung, die MigrantInnen auch als Produzenten von innerer Sicherheit und AkteurInnen in Konfliktlösungsprozessen sehen. Auch dieser Beitrag setzt sich kritisch mit zentralen administrativen Kategorisierungen auseinander. In der Tradition einer sich kritisch verstehenden Kriminologie beanstanden die AutorInnen die mangelnde Reflexion bestehender polizeilicher und kriminologischer Migrationsforschung über die legislativen, judikativen und exekutiven Praktiken der Migrationsregulierung. Eine Forschung, die Migration nicht als ,administrative Kategorie“ in den Blick nimmt, verunmöglicht eine kritische Reflexion über die Generierung von Wissen aus Rechts- und polizeilichen Praxen, die auf Begriffe wie ,(Il-)Legalität“ und „Kriminalitätsbelastung“ von MigrantInnen gründen. Ein Perspektivenwechsel in dieser Forschungstradition würde den Fokus weniger auf „Kriminalität“ oder „Kriminelle“ setzen, sondern jene kriminaljustiziellen Institutionen untersuchen, die bestimmte Handlungen oder Personen(gruppen) so definieren.

Der Aufsatz von Winfried Moser, Korinna Lindinger und Caterina Hannes „Früher Schulabgang in Österreich“ hinterfragt die Kategorie „Migrationshintergrund“ hinsichtlich ihres Beitrags zur Erklärung des Schulausstiegsrisikos von 15- bis 19jährigen Jugendlichen in Österreich. Ausganspunkt der Analyse sind empirische Belege über das erhöhte Bildungsausstiegrisiko migrantischer Jugendlicher, das mehr 
als dreimal so hoch erscheint wie bei Gleichaltrigen ohne Migrationshintergrund. Der Beitrag setzt sich zum Ziel, die Erklärungskraft dieses über Fremdzuschreibung gemessenen Individualmerkmals zu überprüfen, indem er den Einfluss eines weiterführenden Angebots nach der Pflichtschule auf das Risiko für einen frühen Schulabgang analysiert und dabei zeigt, dass das Distinktionsmerkmal „Migrationshintegrund" nur scheinbar mit dem Schulausstiegsrisiko korreliert. Zudem zeigen die Ergebnisse, dass ein verstärktes Augenmerk auf die Situation jener Jugendlichen gerichtet werden muss, die ihre Pflichtschulzeit nur zum Teil oder gar nicht in Österreich verbracht haben, und dass ein stark auf die Lehrausbildung konzentriertes regionales Bildungsangebot vor Ausbildungsabbruch schützt. Allerdings ist dieser Schutz selektiv und kommt allein autochthonen Jugendlichen zugute.

\subsection{Forschung als Prozess von (Un-)Sichtbarmachung}

Die Unsichtbarkeit spezieller Kategorien insbesondere von Drittstaatsangehörigen in administrativen und behördlichen Statistiken wird seit einiger Zeit, nicht zuletzt aufgrund kritischer Interventionen der Migrationsforschung, anerkannt. Entsprechende Bemühungen werden vor allem in der Konstruktion einer neuen Kategorie sichtbar, den sogenannten ,hard-to-reach“ oder ,hard-to-identify“-Gruppen ${ }^{3}$. In dieser Kategorie sind alle Bevölkerungsgruppen, die in der behördlichen Statistik unsichtbar (geworden) sind, zusammengefasst: Roma, die sich nicht als Roma definieren, Muslime, Angehörige der zweiten Generation, Eingebürgerte und erfolgreich Integrierte, Wohnungslose und Gestrandete, Undokumentierte und international Mobile, multilokal Verwurzelte und Transnationale, Angehörige von Diasporas - Ausdruck einer gesellschaftlichen Diversität, die sich im doppelten Sinn als illusiv beschreiben lässt: unwirklich, weil es sie statistisch nicht gibt und sie sich der administrativen Vermessung entziehen; trügerisch, weil über sie, vom Anekdotischen oder Literarischen abgesehen, kaum etwas bekannt ist; verborgen und umso mehr den Phantasien und verzerrenden Projektionen ausgeliefert.

Diese Situation konfrontiert die Migrationsforschung mit erheblichen Herausforderungen. Einen Schwerpunkt der migrationssoziologischen Diskussion bilden deshalb methodologische Problemstellungen, etwa in Bezug auf die Qualität der Datengrundlagen, die Definition von Grundgesamtheiten, die Erreichbarkeit von Zielpopulationen oder den Umgang mit Mehrsprachigkeit (vgl. Vargas-Silva 2012). Der Ruf nach Entwicklung und Anwendung adäquater Methodologie zur Erforschung migrationsbedingter gesellschaftlicher Realitäten wird auch von einer gesellschaftskritischen Migrationsforschung gestellt, die die Folgen von Migration nicht losgelöst von den Bedingungen der globalisierten Arbeitsteilung, transnationalen Formen von Lebensführung und Vergesellschaftung und der Eigenlogik migrantischer Handlungsentwürfe und Identitäten, die sich im Widerstand gegen Entrechtung und sozialen Kämpfen herausbilden (vgl. Papadopoulos et al. 2008), untersucht. Die Forde-

\footnotetext{
3 Dieser Trend ist auf nationaler und internationaler Ebene zu beobachten. Vgl. dazu die zunehmende Anzahl von Workshops, die ,hard-to-reach“ oder ,,illusive populations“ thematisieren: http://www. unece.org/stats/documents/2014.10.migration2.html; http://inclusivegrowth.be/events/call9/programmeand-presentations.
} 
rung richtet sich dabei auch auf eine kontinuierliche Reflexion der Bedingungen der Wissensproduktion, einschließlich der eigenen Position als Forscher oder Forscherin. Der Hinweis auf Forschung als Prozess von (Un-)Sichtbarmachung unterstreicht das gesellschaftliche Spannungs- und Konfliktfeld, in dem sich Migrationssoziologie bewegt, welches durch sie geöffnet wird und aus dem heraus vielfältige politische und vor allem auch ethische Dilemmata erzeugt werden, sei es im Zusammenhang mit subversiven Überlebensstrategien, klandestinem Wissen oder der Existenz von Schattenorten (Tazzioli 2015; Sandoval 2013).

Die drei letzten Beiträge des Sonderheftes widmen sich aus unterschiedlichen Forschungszusammenhängen methodologischen Fragen. Der Text „Unveiling what should remain hidden: Ethnics and politics of researching marginal people" von Michal Ruzicka bietet eine Reflexion der Rolle ethnographischer Zugange im Kontext der Beforschung marginalisierter Gruppen wie jene der Roma. Der Autor unterstreicht zunächst die Notwendigkeit einer profunden Kenntnis der staatlichen Rahmenbedingungen für das Verstehen der subjektiven Wirklichkeiten von marginalisierten Personen oder Gruppen. Ruzicka weist auf Problemfelder sozialwissenschaftlicher Forschung hin, die zwar selten im Zentrum einer kritischen Analyse stehen, dem gesamten Forschungsfeld jedoch inhärent sind. Dazu gehören vorrangig die ethischen Dilemmata, in die ForscherInnen geraten, wenn sie einerseits Zugang zu Wissen durch ethnographische Methoden erschließen und es sich andererseits dabei gerade um jene Wissensbestände handelt, deren Enthüllung die Mitteilenden gefährden kann.

Auch der Beitrag von Andrea Fritsche, „Kultur(en) und Sprach(en) der Asylwirklichkeit“, widmet sich einer Analysekategorie der Migrationsforschung, der aus unterschiedlichen methodologischen Gründen bislang nur begrenzte Aufmerksamkeit geschenkt wurde - Flüchtlinge und AsylwerberInnen. Dabei stellen sich für Fritsche zwei grundlegende Fragen: zum einen, wie können hilfs- und schutzbedürftige Personen, die vermutlich Traumatisierungen erfahren haben, im Rahmen sozialwissenschaftlicher Forschung befragt werden; zum anderen, wie ist Verstehen im Kontext von Interkulturalität und Mehrsprachigkeit möglich, beziehungsweise wie können latente Bedeutungen erschlossen werden. Basierend auf der Analyse von rund 30 qualitativen Interviews mit AsylwerberInnen aus 14 unterschiedlichen Herkunftsstaaten, und ergänzt durch teilnehmende Beobachtung und eine Analyse von nicht-reaktiv entstandenen Dokumenten, reflektiert Fritsche über bestehende Herausforderungen der empirischen Forschung, sowohl in Bezug auf Datenerhebung als auch Auswertung. Zudem weist dieser Beitrag auf jene Strukturen und Prozesse der Verrechtlichung und Bürokratisierung hin, die bestimmte Kompetenzen, beziehungsweise Erzähl- und Darstellungsformen seitens der AsylwerberInnen mitreproduzieren. Fritsche zeigt, dass Einvernahmen im Asylprozess durch ein Rollenmissverhältnis gekennzeichnet sind, das zu Negierung von individuellen oder kulturell bedingten Erzählformen und zu Reproduktion von narrativen Ungleichheiten führt.

Julia Dahlvik, deren Beitrag „Asylanträge verwalten und entscheiden“ unser Sonderheft abschließt, gibt einen Einblick auf verborgen gebliebene Prozesse der Migrationsverwaltung, insbesondere auf die alltäglichen Prozesse und sozialen Praktiken, die im Rahmen der Bearbeitung von Asylanträgen im Bundesasylamt stattfinden. 
Trotz kontinuierlicher Reformen und steigender Antragszahlen, und obwohl sie das Leben von immigrierten Personen mitstrukturieren, sind die betreffenden Institutionen empirisch kaum untersucht worden. Indem sich Dahlvik mit zwei in der deutschsprachigen Soziologie wenig beachteten Themenfeldern befasst - zum einen die der Verwaltung von Migration und zum anderen die der Aktenanalyse - werden jene Praktiken, Prozesse, Herausforderungen und Ermessensspielräume sichtbar gemacht, die den Arbeitsalltag der EntscheiderInnen prägen und das Handeln der Institution ausformen. Dahlviks Zugang, der sich durch eine Kombination unterschiedlicher qualitativer Methoden auszeichnet und der unter dem Begriff institutionelle Ethnographie subsumiert werden kann, transzendiert enggezogene fachliche Grenzen und erlaubt damit Einblicke in das „Innenleben“ der betreffenden Institution und in das von Asylakten. Der Beitrag unterstreicht damit auch das Potential vernachlässigter Analysemethoden für die Migrationsforschung, wie jene der Artefakten- bzw. Aktenanalyse.

So wird in den drei abschließenden Beiträgen nochmals und in zugespitzter Weise eine Herausforderung benannt, der sich auch die anderen Beiträge dieses Sonderheft stellen und mit der sich jede migrationssoziologische Forschung direkt oder indirekt konfrontiert sieht: die Mitwirkung an der Produktion von bestimmten Formen gesellschaftlicher Unsichtbarkeit und Irrationalität.

\section{Literatur}

Abel, Guy J., und Nikola Sander. 2014. Quantifying global international migration flows. Science 343(6178):1520-1522.

Amelina, Anna, Thomas Faist, und Devrimsel G. Nergiz (Hrsg.). 2013. Methodologies on the move. The transnational turn in empirical migration research. London: Routledge.

Amelina, Anna, Kenneth Horvath, und Bruno Meeus (Hrsg.). 2015. An interdisciplinary anthology of migration and social transformation: European perspectives. Amsterdam: Amsterdam University Press.

Beck, Ulrich, und Edgar Grande. 2010. Jenseits des methodologischen Nationalismus. Außereuropäische und europäische Variationen der Zweiten Moderne. Soziale Welt 61:187-216.

Brighenti, Andrea. 2007. Visibility. A category for the social sciences. Current Sociology 55(3):323-342.

Carvill Schellenbacher, Jennifer, Julia Dahlvik, Heinz Fassmann, und Christoph Reinprecht (Hrsg.). 2016. Migration und Integration - wissenschaftliche Perspektiven aus Österreich: Jahrbuch 3/2016. Göttingen: Vandenhoeck \& Ruprecht.

Castles, Stephen. 2010. Understanding global migration: a social transformation perspective. Journal of ethnic and migration studies 36(10):1565-1586.

Cvajner, Martina, und Giuseppe Sciortino. 2010. Theorizing irregular migration: the control of spatial mobility in differentiated societies. European Journal of Social Theory 13(3):389-404.

Czaika, Mathias, und Hein de Haas. 2014. The globalization of migration. Has the world become more migratory? International Migration Review 48(2):283-323.

Dahinden, Janine. 2016. Migration im Fokus? Plädoyer für eine reflexive Migrationsforschung. In Migration und Integration - wissenschaftliche Perspektiven aus Österreich: Jahrbuch 3/2016, Hrsg. Jennifer Carvill Schellenbacher, Julia Dahlvik, Heinz Fassmann, und Christoph Reinprecht, 11-32. Göttingen: Vandenhoeck \& Ruprecht.

Dahlvik, Julia, und Christoph Reinprecht. 2015. Asyl als Widerspruch. Vom Menschenrecht zum Auserwählten. In: Jelinek[Jahr]Buch 2014-1015, Hrsg. Pia Janke, 41-52. Wien, Praesens Verlag.

Dahlvik, Julia, Heinz Fassmann, und Wiebke Sievers (Hrsg.). 2012. Migration und Integration - wissenschaftliche Perspektiven aus Österreich: Jahrbuch 1/2011. Göttingen: Vandenhoeck \& Ruprecht.

Dahlvik, Julia, Christoph Reinprecht, und Wiebke Sievers (Hrsg.). 2014. Migration und Integration - wissenschaftliche Perspektiven aus Österreich: Jahrbuch 2/2013. Göttingen: Vandenhoeck \& Ruprecht.

Esser, Hartmut. 1980. Aspekte der Wanderungssoziologie. Assimilation und Integration von Wanderern, ethnischen Gruppen und Minderheiten. Eine handlungstheoretische Analyse. Darmstadt Neuwied: Luchterhand. 
Fassmann, Heinz (Hrsg.). 2007. 2. Österreichischer Migrations- und Integrationsbericht 2001-2006. Rechtliche Rahmenbedingungen, demographische Entwicklungen, sozioökonomische Strukturen. Klagenfurt Celovec: Drava.

Fassmann, Heinz, und Irene Stacher (Hrsg.). 2003. Österreichischer Migrations- und Integrationsbericht 2001-2006. Demographische Entwicklungen, sozioökonomische Strukturen, rechtliche Rahmenbedingungen. Klagenfurt Celovec: Drava.

Fassmann, Heinz, Ursula Reeger, und Wiebke Sievers (Hrsg.). 2009. Statistics and reality. Concepts and measurement of migration in europe. Amsterdam: Amsterdam University Press.

Favell, Adrian. 2014. The fourth freedom: theories of migration and mobilities in „neo-liberal“ Europe. European Journal of Social Theory 17(3):275-289.

Goldin, Ian, Cameron Goffrey, und Meera Balarajan. 2012. Exceptional people. How migration shaped our world and will define our future. Princeton: Princeton University Press.

Hoffmann-Nowotny, Hans-Joachim. 1973. Soziologie des Fremdarbeiterproblems. Eine theoretische und empirische Analyse am Beispiel der Schweiz. Stuttgart: Enke.

Latcheva, Rossalina, und Barbara Herzog-Punzenberger. 2011. integration revisited. Zur Dynamik und Kontextabhängigkeit individueller Integrationsverläufen am Beispiel von MigrantInnen der ersten Generation in Wien. Österreichische Zeitschrift für Soziologie 36(1):3-27.

Martiniello, Marco, und Andrea Rea. 2014. The concept of migratory careers: elements for a new theoretical perspective of contemporary human mobility. Current Sociology 62(7):1079-1096.

Massey, Douglas, Joaquin Arango, Graeme Hugo, Ali Kouaouci, Adela Pellegrino, und Edward J. Taylor. 2008. Worlds in motion: understanding international migration at the end of the millennium. Oxford: Clarendon.

Mecheril, Paul, Susanne Arens, Claus Melter, Oscar Thonas-Olalde, und Elisabeth Romaner (Hrsg.). 2013. Migrationsforschung als Kritik?: Spielräume kritischer Migrationsforschung. Wiesbaden: Springer VS.

O'Reilly, Karen. 2012. Migration and Social Theory. Basingstoke: Palgrave Macmillan.

Papadopoulos, Dimitris, Niamh Stephenson, und Vassilis Tsianos. 2008. Escape routes. Control and subversion in the 21th century. London: Pluto.

Papastergiadis, Nikos. 2010. Wars of mobility. European Journal of Social Theory 13(3):343-361.

Park, Robert E. 1939. The nature of race relations. In Race relations and the race problem, Hrsg. Edgar T. Thompson, 3-45. Durham: Duke University Press.

Perchinig, Bernhard, und Tobias Troger. 2011. Migrationshintergrund als Differenzkategorie. Vom notwendigen Konflikt zwischen Theorie und Empirie in der Migrationsforschung. In Zukunft. Werte. Europa. Die Europäische Wertestudie 1990-2010. Österreich im Vergleich, Hrsg. Regina Polak, 283-319. Wien: Böhlau.

Portes, Alejandro. 2000. Immigration theory for a new century: some problems and opportunities. International Migration Review 31(4):799-825.

Portes, Alejandro. 2010. Migration and social change: some conceptual reflections. Journal of Ethnic and Migration Studies 36(10):1537-1563.

Portes, Alejandro, und Joseh DeWind (Hrsg.). 2007. Rethinking migration. New theoretical and empirical perspectives. New York: Berghahn.

Rodet, Marie. 2008. Migrants in French Sudan: gender biases in the historiography. In Trans-atlantic migration: the paradoxes of exile, Hrsg. Niyi Afolabi, und Toyin Falola, 165-181. New York: Routledge.

Sandoval, Gerardo Francisco. 2013. Shadow transnationalism: cross-border networks and planning challenges of transnational unauthorized immigrant communitiest. Journal of Planning Education and Research 33(2):176-193.

Skeldon, Ronald. 2012. Going round in circles, circular migration, poverty alleviation and marginality. International Migration 50(3):43-60.

Tazzioli, Martina. 2015. Which Europa? Migrants' uneven geographies and counter-mapping at the limits of representation movements. Journal für kritische Migrations- und Grenzregimeforschung 1(2). http://movements-journal.org/issues/02.kaempfe/04.tazzioli--europe-migrants-geographiescounter-mapping-representation.html. Zugegriffen: 15.07.2016.

Tsianos, Vassilis, und Serhat Karakayali. 2010. Transnational migration and the emergence of the European border regime: an ethnographic analysis. European Journal of Social Theory 13(3):373-387.

Van Haer, Nicholas. 2010. Theories of migration and social change. Journal of Ethnic and Migration Studies 36(10):1531-1536.

Vargas-Silva, Carlos (Hrsg.). 2012. Handbook of research methods in migration. Cheltenham: Edward Elgar. 
Vollmer, Bastian A., Deniz Şenol Sert, und Ahmet İçduygu. 2015. Introduction: Eurocentrism and the field of migration research. Migration and Development 4(2):232-237.

Wimmer, Andreas, und Nina Glick Schiller. 2002. Methodological nationalism and beyond: nation-state building, migration and the social sciences. Global Networks 2(4):301-334.

Yildiz, Erol. 2015. Postmigrantische Perspektiven. Aufbruch in eine neue Geschichtlichkeit. In Nach der Migration. Postmigrantische Perspektiven jenseits der Parallelgesellschaft, Hrsg. Erol Yildiz, und Marc Hill, 16-36. Bielefeld: transcript.

Christoph Reinprecht forscht und lehrt Soziologie an der Universität Wien und ist assoziierter Wissenschaftler am Centre de recherche sur l'habitat in Paris. Seine Forschungsschwerpunkte sind Migration und Integration, städtische Lebenszusammenhänge, politische Soziologie und soziale Ungleichheit. Jüngste Buchpublikation (gemeinsam mit Roland Hsu): Migration and Integration: New Models for Mobility and Coexistence. Göttingen: Vandenhoeck \& Ruprecht 2016.

Rossalina Latcheva ist Expertin für Statistik und Surveyforschung in der Agentur der Europäischen Union für Grundrechte (FRA) in Wien und war zuvor am Soziologischen Institut der Universität Zürich als Universitätsassistentin (Post Doc) tätig. Ihre Forschungsschwerpunkte sind Migration und Integration, Diskriminierung, Fremdenfeindlichkeit und ethnische Exklusion, geschlechtsspezifische Gewalt und mixedmethods. 